\title{
A novel isoquinoline alkaloid, DD-carboxypeptidase inhibitor, with antibacterial activity isolated from Streptomyces sp. 8812. Part II: Physicochemical properties and structure elucidation
}

\author{
Jolanta Solecka $^{1}$, Jerzy Sitkowski ${ }^{2,3}$, Wojciech Bocian ${ }^{2,3}$, Elżbieta Bednarek ${ }^{2}$, Robert Kawęcki ${ }^{3}$ and \\ Lech Kozerski ${ }^{2,3}$
}

A novel antimicrobial agent labeled JS-1, being a member of isoquinoline alkaloids, of molecular formula $\mathrm{C}_{10} \mathrm{H}_{9} \mathrm{NO}_{4}$ was isolated from the culture broth of Streptomyces sp. 8812. In this study, we present the structure based on physicochemical and spectroscopic NMR investigations and on quantum chemical structure modeling. The structure of a molecule suggests the biosynthetic path starting from 3 '-hydroxy tyrosine. The synthesis was undertaken and it resulted in NMR data that fully agree with the presented analysis.

The Journal of Antibiotics (2009) 62, 581-585; doi:10.1038/ja.2009.86; published online 28 August 2009

Keywords: antibacterial activity; DD-peptidase inhibitor; isoquinoline alkaloid; JS-1 structure; NMR

\section{INTRODUCTION}

The increasing bacterial resistance to antibiotics causes a great therapeutic problem because of the widespread presence of multiresistant pathogenic bacteria. The solution of this problem involves screening for microorganisms producing novel antimicrobial drugs. Streptomycetes are well-known producers of antibiotic and other bioactive metabolites. ${ }^{1}$ The purpose of this work was the investigation of novel antimicrobial agents, inhibitors of DD-carboxypeptidase/transpeptidase (DD-peptidase). In our screening program for new inhibitors of DD-peptidase 64-575 II (EC 3.4.16.4) ${ }^{2-4}$ from microbial secondary metabolites, a novel isoquinoline alkaloid with documented antibacterial activity, JS-1, was isolated from the culture broth of Streptomyces sp. $8812 .{ }^{5}$ In a recent paper, ${ }^{6}$ we have described the taxonomy, fermentation of producing strain and isolation, as well as the purification and biological activity of novel isoquinoline alkaloid. In this study, we present the structure based on physicochemical and spectroscopic NMR investigations, quantum chemical structure modeling and synthesis protocol, which confirmed an established structure and chirality.

\section{RESULTS AND DISCUSSION}

The physicochemical properties of a compound are shown in Table 1.

JS-1 is soluble in water (phosphate buffer $\mathrm{pH}$ 7.2), and insoluble in $\mathrm{CHCl}_{3}$ or acetonitrile. It is soluble, but degrading, in methanol and dimethylosulfoxide.
The molecular formula of JS-1 was determined to be $\mathrm{C}_{10} \mathrm{H}_{9} \mathrm{NO}_{4}$ on a basis of high resolution ESI-MS $\left[(\mathrm{M}+\mathrm{H})^{+}, 208.06043 \mathrm{~m} / \mathrm{z}\right.$ $(-0.03 \mathrm{mmu}$ error $)]$, thus requiring 7 degrees of unsaturation. The IR spectrum of JS-1 showed a broad hydroxyl band at $3436 \mathrm{~cm}^{-1}$, and the multiple bands in a region of $1600-1700 \mathrm{~cm}^{-1}$ may indicate the presence of an aromatic carbonyl group, a $\mathrm{C}=\mathrm{N}$ double bond at $1681 \mathrm{~cm}^{-1}$, a carboxylate group as well as a carbonyl group conjugated to a double bond, or an enamine at the C-N bond at $1636 \mathrm{~cm}^{-1}$. The UV absorption maximum at $321 \mathrm{~nm}$ suggests the presence of a conjugated system.

JS led us to suggest an isoquinoline structure shown in Figure 1.

It is essential to know which tautomeric form has the biological activity and therefore all spectroscopic investigations were carried out in physiological conditions, that is, $\mathrm{pH} 7.2$ in phosphate buffer. The tautomeric equilibrium, if present, is fast in the NMR timescale and therefore only one set of sharp signals is observed at room temperature in buffered water solution. In Table 2, NMR data are presented that allow the confirmation of a structure.

A deceptively simple ${ }^{1} \mathrm{H}$ NMR spectrum, consisting of only three singlets, at 6.53, 7.04 and 8.23 p.p.m., and an ABM system, at 4.39, 3.29 and 3.18 p.p.m., with 5 heteroatoms present makes structure assignment ambiguous and requires a detailed recourse to ${ }^{1} \mathrm{H} \sim{ }^{1} \mathrm{H}$ and ${ }^{1} \mathrm{H} \sim{ }^{13} \mathrm{C}$ correlations in heteronuclear single quantum coherence (HSQC), heteronuclear multiple bond correlation (HMBC) and

${ }^{1}$ Independent Laboratory of Streptomycetes and Fungi Imperfecti, National Institute of Public Health—National Institute of Hygiene, Warsaw, Poland; ${ }^{2}$ National Medicines Institute, Warsaw, Poland and ${ }^{3}$ Institute of Organic Chemistry, Polish Academy of Sciences, Warsaw, Poland

Correspondence: Dr J Solecka, Independent Laboratory of Streptomycetes and Fungi Imperfecti, National Institute of Public Health-National Institute of Hygiene, Chocimska 24, Warsaw 00-791, Poland.

E-mail: jsolecka@pzh.gov.pl

Received 7 May 2009; revised 9 June 2009; accepted 11 June 2009; published online 28 August 2009 
double pulsed field gradient spin-echo NOE, ${ }^{7}$ respectively. In addition, dynamic $\mathrm{OH}, \mathrm{COOH}$ and $\mathrm{NH}$ protons are in fast exchange with bulk water, thus making impossible the assignment of nitrogen atom functionality in the latter case, by means of running the ${ }^{1} \mathrm{H}_{-}{ }^{15} \mathrm{~N}$ HSQC spectrum or drastically less sensitive ${ }^{1} \mathrm{H}-{ }^{15} \mathrm{~N}$ HMBC correlation.

${ }^{13} \mathrm{C}$ Chemical shifts of proton-bearing carbon atoms were established using the HSQC spectrum. It proved useful to distinguish two carbon atoms of the same chemical shifts, that is, C-5 and C-8, 117.1 p.p.m., and to assign the two geminal protons to carbon atom C-4 at 3.29 and 3.18 p.p.m. This latter observation brings about the important conclusion that the molecule has a stable stereogenic center giving rise to the nonequivalence of the geminal protons. The ${ }^{3} \mathrm{~J}$ $(\mathrm{H}, \mathrm{H})$ coupling constants in the ABM system of 7.4 and $9.2 \mathrm{~Hz}$ indicate that the stereogenic center is in vicinal arrangement with the geminal protons. Figure 2 presents key correlations split for clarity into two projections. Only one tautomer, ketone, is shown for simplicity. The HMBC correlations in a left projection prove the connection of an AMX aliphatic system to aromatic carbon atom C-5. The NOE

Table 1 Physicochemical properties of JS-1

\begin{tabular}{ll}
\hline Appearance & Yellow powder \\
\hline$[\alpha]_{D}^{23}$ & $21.1^{\circ}\left(c=0.185, \mathrm{H}_{2} \mathrm{O}\right)$ \\
Molecular formula & $\mathrm{C}_{10} \mathrm{H}_{9} \mathrm{NO}_{4}$ \\
LR ESI-MS $(\mathrm{m} / \mathrm{z})$ & \\
$\quad$ & $208(\mathrm{M}+\mathrm{H})^{+}$ \\
$\quad$ Positive & $206(\mathrm{M}-\mathrm{H})^{-}$ \\
$\quad$ Negative & \\
HRESI-MS $(m / z)$ & 208.06043 \\
$\quad$ Calcd & 208.06040 \\
$\quad$ Found $(\mathrm{M}+\mathrm{H})^{+}$ & $397.92(3475), 321.33(943), 266.38(2225)$ \\
UV $\lambda_{\max } \mathrm{nm}(\varepsilon)\left(\mathrm{H}_{2} \mathrm{O}\right)$ & $3436,1681,1636,1131,1075,986,984,804,724$ \\
IR $(\mathrm{KBr}) v_{\max } \mathrm{cm}^{-1}$ &
\end{tabular}

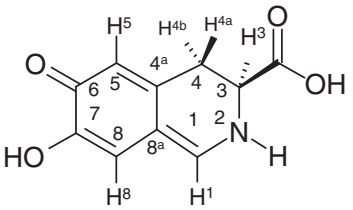<smiles>[1H]c1c(O)c(O)c(P)c2c1C=N[C@H](C(=O)O)[C@@H]2[18F]</smiles>

Figure 1 Postulated structure of JS-1. contacts also prove the spatial vicinity of $1-\mathrm{H}$ and $8-\mathrm{H}$, both being distant from 5-H. Comparable NOE effects in both directions point to the isolated two-spin system, in agreement with the structural environment. The NOE contacts in the right projection in Figure 2 confirm the connectivity of $5-\mathrm{H}$ with the ABM system. In addition, nearly equal NOEs from $\mathrm{H}-5$ to $\mathrm{H} 4^{\mathrm{a}}$ and $\mathrm{H}-4^{\mathrm{b}}$ suggest that they are nearly equidistant to $5-\mathrm{H}$. Three-bond $\mathrm{HMBC}$ correlations from $8-\mathrm{H}$ to $\mathrm{C}-1$ and $\mathrm{C}-6$ and from 1-H to $\mathrm{C}-3$ allow for the unambiguous assignment of $\delta_{\mathrm{C}} 159.5$ and 168.8 p.p.m. to their positions in a structure, C- 1 and C-6, respectively. Our experience in an enamine carbon chemical shifts suggests that the same assignment as $=\mathrm{C}-\mathrm{N}$ carbon atom with a formally single bond in a conjugated system of $\alpha, \beta$-unsaturated ketones gives resonances up to 155 p.p.m. for an alkyl disubstituted nitrogen atom. ${ }^{8}$

In contrast, the $\mathrm{C}=\mathrm{N}$ bonds in imines give carbon resonances at approximately 160 p.p.m. ${ }^{9,10}$

It is commonly recognized that aromatization leads to enhanced stability and defines the thermodynamically more stable species in equilibrium.

To address in more depth the position of tautomeric equilibrium, we have performed the theoretical calculations of the ${ }^{1} \mathrm{H},{ }^{13} \mathrm{C}$ chemical shift and the geometry of all possible tautomers (see Supplementary Figure 2S) using the PCM protocol in the $\mathrm{H}_{2} \mathrm{O}$ box at the B3LYP/6-31/ $+6\left(2 \mathrm{~d}_{18}\right)$ basis set level. The chemical shift data are compiled in Supplementary Tables 1S-3S in the Supplementary materials along with the structures. Scarce data regarding the ${ }^{13} \mathrm{C}$ chemical shifts in a similar system are available in the literature. ${ }^{12}$

The calculated chemical shifts of conformers suggested for the equilibrium are shown in Figure 3. The protonated dihydroxy imine tautomer $4 \mathbf{a}$ can also be considered as a species present in equilibrium.

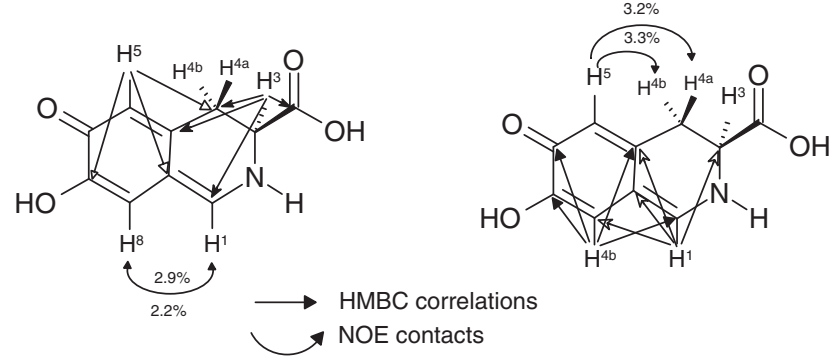

Figure 2 Key HMBC correlations and $\mathrm{NOE}^{11}$ contacts in JS-1. For clarity, only one tautomer is shown.

Table $2{ }^{13} \mathrm{C}(176 \mathrm{MHz})$ and ${ }^{1} \mathrm{H}(500 \mathrm{MHz})$ NMR data for JS-1 in buffered water solution (pH 7.2)

\begin{tabular}{|c|c|c|c|c|}
\hline \multicolumn{5}{|c|}{ Proton spectral parameters, scalar, ${ }^{1} \mathrm{H}-{ }^{13} \mathrm{C}$, and dipolar, ${ }^{1} \mathrm{H}-{ }^{1} \mathrm{H}$, correlations } \\
\hline C & $\delta_{C}$ & $\delta_{H}$ & HMBC correlations & DPFGSE NOE contacts (\%) \\
\hline 1 & 159.5 & $8.21(\mathrm{~s})$ & $\mathrm{C}-3, \mathrm{C}-4 \mathrm{a}, \mathrm{C}-8, \mathrm{C} 8 \mathrm{a}$ & $8-\mathrm{H}(2.9)$ \\
\hline 3 & 55.8 & $4.39(\mathrm{dd}, 7.4,9.2)$ & $C-1, C-4, C-4 a, C=0$ & $4-\mathrm{H}^{\mathrm{a}}(1.7) ; 4-\mathrm{H}^{\mathrm{b}}(1.1)$ \\
\hline 4 & 29.2 & $3.29(\mathrm{dd}, 7.4,16.7) 4-\mathrm{H}^{\mathrm{a}} 3.18(\mathrm{dd}, 9.2,16.7) 4-\mathrm{H}^{\mathrm{b}}$ & $C-3, C-4 a, C-8 a, C=0 \quad C-3, C-4 a$ & $3-\mathrm{H}(1.9) ; 5-\mathrm{H}(1.5)^{*}$ \\
\hline $4 a$ & 135.0 & & & \\
\hline 5 & 117.1 & $6.53(\mathrm{~s})$ & C-4, C-7, C-8a & $4-\mathrm{H}^{\mathrm{a}}(1.1) ; 4-\mathrm{H}^{\mathrm{b}}(1.2)$ \\
\hline 6 & 168.8 & & & \\
\hline 7 & 146.5 & & & \\
\hline 8 & 117.1 & 7.04(s) & & $1-\mathrm{H}(2.2)$ \\
\hline $8^{\mathrm{a}}$ & 110.5 & & & \\
\hline
\end{tabular}

Abbreviations: DPFGSE NOE, double pulsed field gradient spin-echo NOE; HMBC, heteronuclear multiple bond correlation.

*These values represent the effect of simultaneous irradiation of both $4-\mathrm{H}^{\mathrm{a}}$ and $4-\mathrm{H}^{\mathrm{b}}$ protons, which cannot be selectively irradiated due to strong coupling 


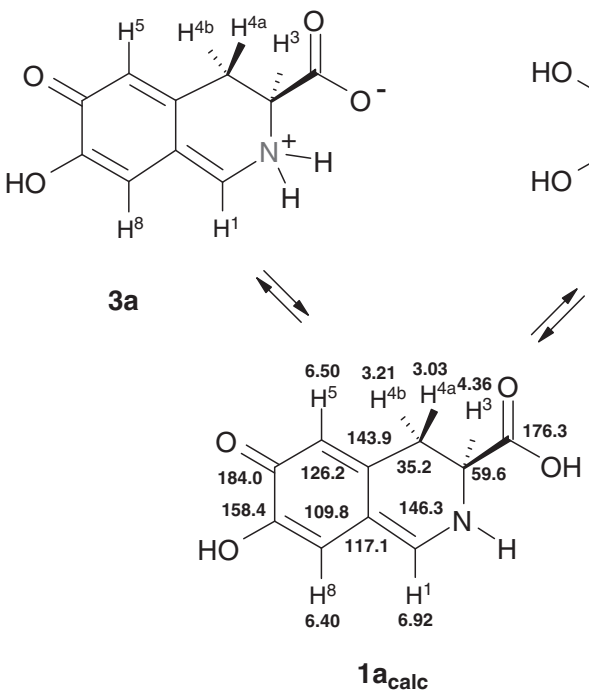<smiles>[2H]c1c(O)c(O)cc2c1C=N[C@H](C(=O)O)[C@@H]2[18OH]</smiles>

$2 a$
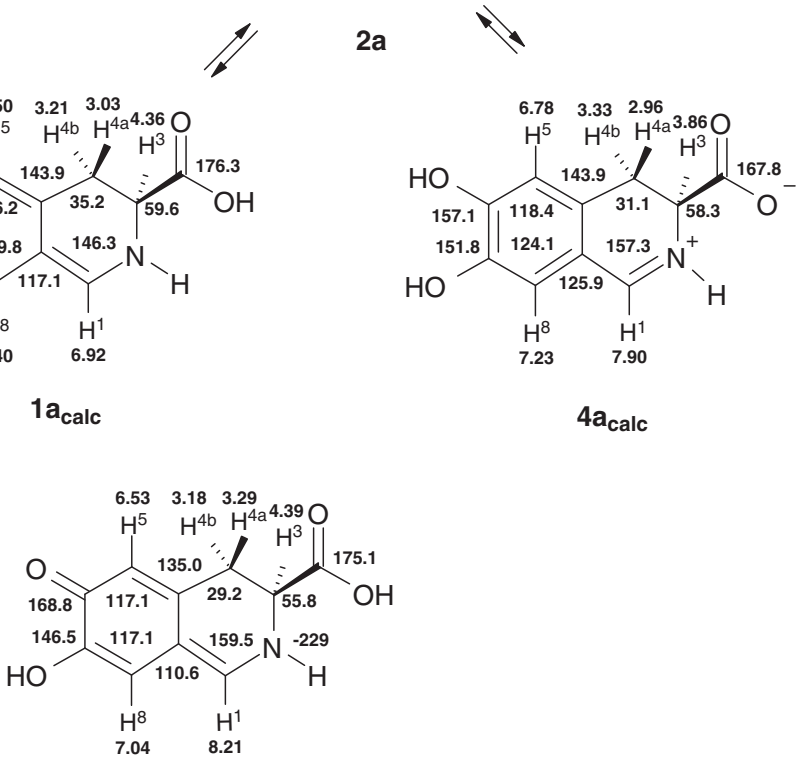

${ }^{1} \mathrm{H},{ }^{13} \mathrm{C}_{\text {exp }}$

Figure 3 Comparison of calculated, in vacuo, ${ }^{1} \mathrm{H}$ and ${ }^{13} \mathrm{C}$ chemical shift in tautomers $\mathbf{1 a}$ and $\mathbf{4 a}$ of $\mathbf{J S}-\mathbf{1}$ with experimental ones, ${ }^{1} \mathbf{H},{ }^{13} \mathbf{C}_{\text {exp }}$, assigned to specific carbon atoms using heteronuclear multiple bond correlation and double pulsed field gradient spin-echo NOE data. The hypothetical structures $2 \mathrm{a}$ and $\mathbf{3 a}$ are also shown (see text).

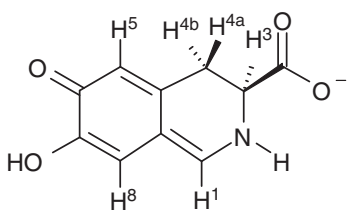

$5 a$

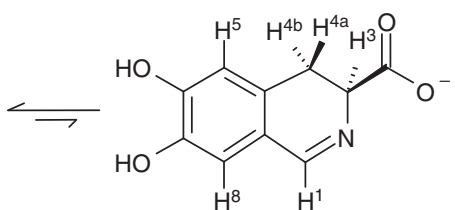

$6 a$
Figure 4 Proposed equilibrium for JS-1 at basic conditions.

The predominance of the enamine tautomer in solution is further confirmed in ${ }^{15} \mathrm{~N}$ NMR, which gives the $\delta\left({ }^{15} \mathrm{~N}\right)=-229.0$ p.p.m. (versus external $\mathrm{CH}_{3} \mathrm{NO}_{2}$ ), at $\mathrm{pH}$ 7.2. This chemical shift is representative both for nitrogen atoms in enamine, $\mathbf{1 a}$ or $\mathbf{1} \mathbf{b}$, and protonated imine (zwitter ion) 4a. A small amount of the form $\mathbf{2 a}$ can be allowed in equilibrium with 1a. The $\mathbf{3 a}$ form cannot be considered on the basis of the observed ${ }^{15} \mathrm{~N}$ chemical shift as its nitrogen resonance appears at approximately -300 p.p.m. from nitromethane. The situation above applies to experimental conditions close to the isoelectric point.

Under more basic conditions, the carboxylate is deprotonated and the equilibrium shown in Figure 4 applies. As discussed above, the ${ }^{15} \mathrm{~N}$ chemical shift allows a small amount of imine in equilibrium.

It is worth noting that near the isoelectric point, $\mathrm{pI}=6.0$, for this compound (see to Supplementary Materials), the molecule can be both neutral and ionized and therefore the species discussed in Figures 3 and 4 should be considered.
Figure 5 presents the possible species in tautomeric equilibrium in solution. Structures $\mathbf{1 a}$ and $\mathbf{1} \mathbf{b}$ are the conformers having planar ring $\mathrm{B}$ in $\mathbf{1 a}$ and twisted in $\mathbf{1} \mathbf{b}$.

The calculations carried out also allow comparison of the geometry of both tautomers, relating it to experimental observables. The geometry of the $\mathrm{N}-\mathrm{C}_{\mathrm{sp} 3}-\mathrm{C}_{\mathrm{sp} 3}$ motif is expected to be most sensitive to tautomerization because of the changes in nitrogen hybridization and bond length around it. The ABM system of investigated molecule offers an experimental check of the calculated geometry along the C4-C3 bond in both conformers shown in Figure 5.

It is shown in Figure 5 that the $\mathrm{H}-4^{\mathrm{a}}$ and $\mathrm{H}-4^{\mathrm{b}}$ protons in $\mathbf{1 b}$ are both in synclinal positions with respect to the $\mathrm{H}-3$ proton, having torsion angles of $-69.9^{\circ}$ and $+47.0^{\circ}$, respectively. This spatial arrangement leads to ${ }^{3} \mathrm{~J}\left(\mathrm{H}-3, \mathrm{H}-4^{\mathrm{a}}\right)$ and ${ }^{3} \mathrm{~J}\left(\mathrm{H}-3, \mathrm{H}-4^{\mathrm{b}}\right)$ vicinal coupling constants 1.6 and $7.7 . \mathrm{Hz}$, in disagreement with observed experimental observation, 7.4 and $9.2 \mathrm{~Hz}$. On the contrary, in $1 \mathrm{a}, \mathrm{H}-4^{\mathrm{b}}$ is in synclinal and $\mathrm{H}-4^{\mathrm{a}}$ in anti-periplanar position to $\mathrm{H}-3$ with torsion angles of $-51.0^{\circ}$ and $-167.0^{\circ}$ leading to 4.5 and $13.7 \mathrm{~Hz}$, respectively, again in disagreement with experimental values. Averaging of the respective coupling constants in both forms results in agreement with the experiment and this fact gives strong evidence to the predominance of this tautomeric form in equilibrium in solution.

With respect to the above discussion, Lakhlifi et al. ${ }^{13}$ report the ${ }^{1} \mathrm{H}$ NMR spectrum of closely related 3,4-dihydro-6,7-dimethoxy-3methylcarboxylate-isoquinoline. Unfortunately, the geminal nonequivalence of $\mathrm{H}-4^{\mathrm{a}}$ and $\mathrm{H}-4^{\mathrm{b}}$ was not observed and the reported splitting in the $\mathrm{A}_{2} \mathrm{M}$ system is $10.2 \mathrm{~Hz}$. 


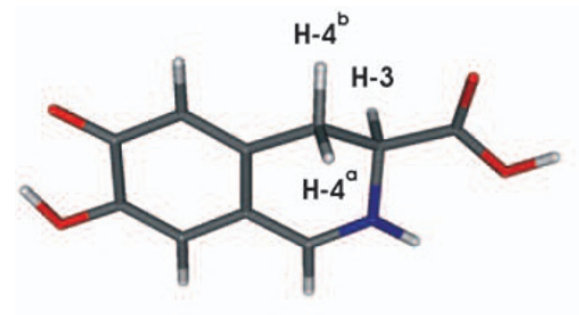

la

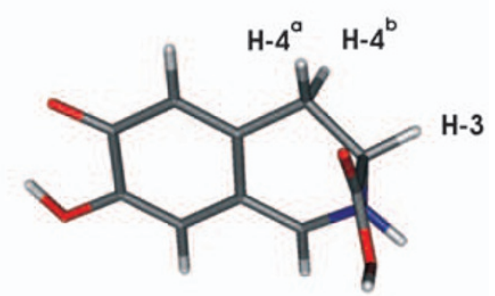

1b

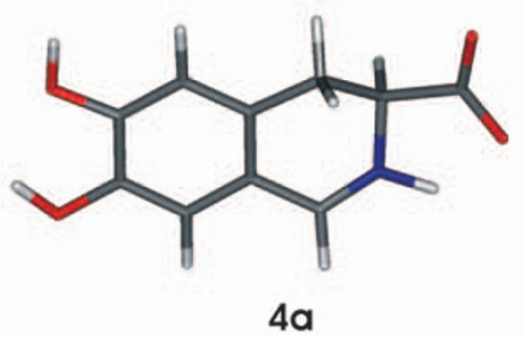

Figure 5 Established structure of JS-1 based on a comparison of calculated and experimental ${ }^{1} \mathrm{H}$ and ${ }^{13} \mathrm{C}$ chemical shifts. Projection along the C4-C3 bond in both conformers $1 \mathrm{a}, 1 \mathrm{~b}$ calculated using the PCM protocol.<smiles>COC(=O)C(N)Cc1ccc(O)c(O)c1</smiles>

a) b)<smiles>O=C(O)[C@H]1Cc2cc(O)c(O)cc2C=[NH+]1</smiles>

a) $\mathrm{HCHO}, \mathrm{Ac}_{2} \mathrm{O}$ b) $\mathrm{POCl}_{3}$

Scheme 1

Finally, the configuration of the C-3 carbon atom should be addressed. The structure of a molecule suggests the biosynthetic path starting from the $3^{\prime}$-hydroxy tyrosine. This defines the (S) C-3 configuration of natural L-amino acid. The verification of this hypothesis was performed by comparison of a specific rotation with the synthetic product.

The synthesis was undertaken and its product yielded NMR data that fully agree with the above analysis (Scheme 1). Spectrum 1S (Supplementary Information, Figure 1S) presents the heteronuclear multiple bond correlation trace of a synthetic product which verifies proper structure elucidation of JS-1.

\section{EXPERIMENTAL SECTION}

IR spectrum was obtained on an FT-IR-1600 Perkin-Elmer spectrophotometer (Perkin-Elmer, Ramsey, MN, USA). UV spectrum was recorded on a Jasco V-630 UV-Visible spectrophotometer (Jasco, Tokyo, Japan). The pI was established using this facility for UV measurements and the Orion $\mathrm{pH}$ meter (Orion, Boston, MA, USA).

The optical rotation was measured with a Jasco P-2000 digital polarimeter. A low-resolution mass spectrum was recorded on an HPLC-MS (Hewlett-Packard 1100; API 365, PESCIEX) mass spectrometer (Hewlett-Packard, Santa Clara, CA, USA). A high-resolution mass spectrum was recorded on a Bruker APEX-Q (9.4T) spectrometer (ESI), positive-ion mode (Bruker, Leipzig, Germany).

The NMR spectra were recorded at $303 \mathrm{~K}$ on a Varian INOVA 500 spectrometer (Varian, Palo Alto, CA, USA) operated at 499.8, 125.7 and $50.51 \mathrm{MHz}$ for ${ }^{1} \mathrm{H}$ and ${ }^{13} \mathrm{C}$, and $15 \mathrm{~N}$, respectively. A $0.1 \mathrm{mg}$ amount of compound was dissolved in $0.7 \mathrm{ml}$ of $\mathrm{H}_{2} \mathrm{O} / \mathrm{D}_{2} \mathrm{O}$ (9:1), $\mathrm{pH} 7.2$ and transferred to a $5 \mathrm{~mm}$ NMR tube. Chemical shifts, $\delta$ given in p.p.m., were referenced against internal reference (trimethylsily1)propanoic acid. The spectrometer was equipped with an inverse ${ }^{1} \mathrm{H}\left\{{ }^{31} \mathrm{P}-{ }^{15} \mathrm{~N}\right\}$, the $5 \mathrm{~mm}$ Nalorac probe with an actively shielded z-gradient coil (ID-pulsed field gradients).

${ }^{1} \mathrm{H}$ NMR was run using the standard method of presaturation of residual water implemented in Varian software.

HSQCAD spectra: ${ }^{14}$ The Echo-antiecho phase-sensitive ${ }^{1} \mathrm{H}-{ }^{13} \mathrm{C}$ HSQC (adiabatic version) NMR spectra were obtained with a spectral width of $6000 \mathrm{~Hz}, 2048$ points in the ${ }^{1} \mathrm{H}$ dimension and $20100 \mathrm{~Hz}, 400$ increments in the ${ }^{13} \mathrm{C}$ dimension; 128 transients per $\mathrm{t}_{1}$ increment, with a relaxation delay of $1.0 \mathrm{~s}$ and ${ }^{1} \mathrm{~J}(\mathrm{C}, \mathrm{H})=135 \mathrm{~Hz}$. The data were linearly predicted to 1600 points and zero-filled to 8192 points in $\mathrm{F}_{1}$ before Fourier transformation.

The ${ }^{1} \mathrm{H} /{ }^{13} \mathrm{C}-\mathrm{HMQC}$ (heteronuclear multiple quantum coherence) technique with pulsed field gradients coherence selection using two pulsed field gradient pulses of relative amplitudes of $+(\gamma \mathrm{I}+\gamma \mathrm{S}):-(\gamma \mathrm{I}-\gamma \mathrm{S})$ and $-(\gamma \mathrm{I}-\gamma \mathrm{S}):+(\gamma \mathrm{I}+\gamma \mathrm{S})$, for heteronuclear echo and antiecho, respectively was used. The S-spin 90 degree pulse low-pass filter was used to suppress one-bond correlation. Such implementation enabled us to obtain a nearly pure absorption line shape along $\mathrm{F}_{1}$, combined with an absolute value mode in $F_{2}$ dimension. ${ }^{15,16}$ The ${ }^{1} \mathrm{H}\left\{{ }^{13} \mathrm{C}\right\}$ HMQC spectra were recorded with the following experimental conditions: an acquisition time of $0.2 \mathrm{~s}$, spectral windows of $5000\left(F_{2}\right)$ and $25500\left(\mathrm{~F}_{1}\right) \mathrm{Hz}$, 2048 data points in the ${ }^{1} \mathrm{H}$ dimension, 256 increments in the ${ }^{13} \mathrm{C}$ dimension, ${ }^{n} J(\mathrm{C}, \mathrm{H})=8 \mathrm{~Hz}$, a $1.3 \mathrm{~s}$ relaxation delay and 512 transients per increment. The data were zero filled to 4096 points in $F_{1}$ and processed using sine bell multiplication in $t_{2}$ and cosine window function in $t_{1}$ dimensions before Fourier transformation. The proton and carbon $\pi / 2$ pulse lengths were 8.0 and $11.5 \mu$ s, respectively.

${ }^{1} \mathrm{H} /{ }^{15} \mathrm{~N}-\mathrm{HMQC}{ }^{1} \mathrm{~N}$ parameters: $\mathrm{F}_{2}, 5000 \mathrm{~Hz} ; \mathrm{F}_{1}, 10000$; number of increments, 80; 512 scans/increment; delay, $120 \mathrm{~ms}$. A low-pass filter (see above) was not used.

Double pulsed field gradient spin-echo NOE experiments were run using a pulse sequence published by Stott et al. ${ }^{17}$ using shaped selective pi soft pulses generated by a standard Varian program. The rsnob pi pulse was calibrated for each individual multiplet.

(S)-6,7-dihydroxy-3,4-dihydroisoquinolin-3-carboxylic acid synthesis: To a warm solution of $N$-formyl-(L)-DOPA ${ }^{18}(70 \mathrm{mg}, 0.31 \mathrm{mmol})$ in acetonitrile $(13 \mathrm{ml})$ was added phosphorus oxychloride $(342 \mathrm{mg}, 2.24 \mathrm{mmol})$ and the mixture was heated under reflux for $1 \mathrm{~h}$. The precipitate was separated and the filtrate was evaporated to give brown oil. The product was purified by preparative HPLC on an RP C18 column (Nucleosil, Duren, Germany) using first $0.1 \%$ TFA in water as eluent and then $10 \%$ acetonitrile in $0.1 \%$ aqueous TFA. Spectral data is identical as for JS-1. 
HRESI-MS calculated for $\mathrm{C}_{10} \mathrm{H}_{10} \mathrm{NO}_{4}(\mathrm{M}+\mathrm{H})^{+}$, 208.06043. Found 208.06093

\section{ACKNOWLEDGEMENTS}

We gratefully acknowledge running the ${ }^{1} \mathrm{H} /{ }^{13} \mathrm{C}-\mathrm{HMBC}$ spectrum at $176 \mathrm{MHz}$ on the limited amount of a sample $(0.03 \mathrm{mg})$ by Prof W Koźmiński, Department of Chemistry, Warsaw University. We also thank Associate Prof Vladimir Havliček, Institute of Microbiology, Laboratory of Molecular Structure Characterization, Prague Czech Republic, for HRESI-MS data.

1 Bérdy, J. Bioactive microbial metabolites. J. Antibiot. 58, 1-26 (2005).

2 Kurzątkowski, W., Solecka, J., Filipek, J., Kurzatkowski, J. D. \& Kurylowicz, W. Streptomycetes excreting DD-carboxypeptidases. Appl. Microbiol. Biotechnol. 33, 452-454 (1990).

3 Solecka, J. \& Kurzatkowski, W. Affinity of exocellular DD-carboxypeptidase/transpeptidase from Saccharopolyspora erythraea PZH 64-575 to beta-lactam compounds. Med. Dosw. Mikrobiol. 51, 151-165 (1999).

4 Solecka, J., Łysek, R., Furman, B., Chmielewski, M. \& Kurzątkowski, W. Practical use of DD-peptidase $64-575$ for assay inhibition activity of natural and synthetic $\beta$-lactam compounds. Acta. Poloniae. Pharmaceutica. 60, 115-118 (2003).

5 Solecka, J. \& Kozerski, L. A new inhibitor of DD-peptidase and its use as antibiotic or anticancer drug. PCT/WO 2009/028972 A1. 5 March (2009).

6 Solecka, J., Rajnisz, A. \& Laudy, A. E. A novel isoquinoline alkaloid, DD-carboxypeptidase inhibitor with antimicrobial activity isolated from Streptomyces sp. 8812. Part I Taxonomy, fermentation, isolation and biological activities. J. Antibiot. (2009) (e-pub ahead of print 28 August 2009; doi:10.1038/ja.2009.85).

7 Stott, K. Stonehouse, J., Keeler, J., Hwang, T.-L. \& Shaka, A. J. Excitation sculpting in high resolution nuclear magnetic resonance spectroscopy: application to selective NOE experiments. J. Am. Chem. Soc. 117, 4199-4200 (1995).
8 Dąbrowski, J., Kamieńska-Trela, K. \& Kozerski, L. Conformational studies by NMR. Part VII. The direct observation of rotational isomerism in some enamino carbonyl compounds and their thione derivatives by CMR. Org. Mag. Reson. 6, 499 (1974).

9 Kozerski, L. \& von Philipsborn, W. Solvent induced deuterium effects in ${ }^{13} \mathrm{C}$ and ${ }^{15} \mathrm{~N}$ NMR spectra of enaminones. Helv. Chim. Acta 65, 2077 (1982)

10 Kozerski, L. et al. The tautomeric equilibrium and stereochemistry of $\beta$-sulfonylenamines. N. J. Chem. 26, 1060-1069 (2002).

11 Neuhaus, D. \& Williamson, M. P. Nuclear Overhauser Effect in Conformational and Structural Analysis. A Guide for Chemists (Viley-VCH Publisher, Inc New York, 1995).

12 Haraguchi, Y., Kozima, S. \& Yamaguchi, R. An efficient assymetric synthesis of alloand pseudo-7,8-dimethoxyberbane systems through Tin-mediated three component coupling. Tetrahedron: Asymmetry 7, 443-449 (1996).

13 Lakhlifi, T., Sedqui, A., Laude, B., Dinh An, N. \& Verbel, J. Cycloaddition d'ylures d'azométhine derives de la 3,4-dihydro-6,7-diméthoxycarbonyl-isoquinoléine. Can. J. Chem. 69, 1156-1160 (1991).

14 Summers, M. F., Marzilli, L. G. \& Bax, A. Complete proton and carbon-13 assignments of coenzyme B12 through the use of new two-dimensional NMR experiments. J. Am. Chem. Soc. 108, 4285-4294 (1986).

15 Meissner, A. \& Sørensen, O. W. Economizing spectrometer time and broadband excitation in small-molecule heteronuclear NMR correlation spectroscopy. Broadband HMBC Magn. Res Chem. 38, 981-984 (2000).

16 KoŸmiñski, W., Bednarek, E., Bocian, W., Sitkowski, J. \& Kozerski, L. J. The new HMQC-based technique for the quantitative determination of heteronuclear coupling constans. Application for the measurement of ${ }^{3} \mathrm{~J}\left(\mathbf{H}_{i}^{\prime}, \mathbf{P}_{i+1}\right)$ in DNA oligomers. Magn. Reson. 160, 120 (2003).

17 Stott, K., Stonehouse, J., Keeler, J., Hwang, T.-L. \& Shaka, A. J. Excitation sculpting in high resolution nuclear magnetic resonance spectroscopy: application to selective NOE experiments. J. Am. Chem. Soc. 117, 4199-4200 (1995).

18 Dickner, T. \& Laschat, S. Pictet-Spengler cyclization vs. aminal formation: competing reaction pathway of Benzo[b][1,7]napthyridines controlled by the configuration. Helv. Chim. Acta 84, 2064-2070 (2001).

Supplementary Information accompanies the paper on The Journal of Antibiotics website (http://www.nature.com/ja) 